\title{
Comparative evaluation of prophylactic single-dose intravenous antibiotic with postoperative antibiotics in elective urologic surgery
}

This article was published in the following Dove Press journal:

Therapeutics and Clinical Risk Management

12 November 2010

Number of times this article has been viewed

Mohammad K Moslemi'

Seyed M Moosavi Movahed ${ }^{2}$

Akram Heidari ${ }^{3}$

Hossein Saghafi ${ }^{2}$

Mehdi Abedinzadeh ${ }^{4}$

'Department of Urology,

2Department of Nephrology,

${ }^{3}$ Department of Health, Kamkar

Hospital, Qom University of Medical

Sciences, Qom, Iran; ${ }^{4}$ Department

of Urology, Moradi Hospital, School

of Medicine, Rafsanjan University of

Medical Sciences, Rafsanjan, Iran
Correspondence: Seyed M Moosavi Movahed

Department of Nephrology, Kamkar Hospital, School of Medicine, Qom University of Medical Sciences, Qom, Iran Tel +989l 21535223

Fax +98 25 |77| 3473

Email moosavimovahed@gmail.com
Background: Unrestricted antibiotic use is very common in Iran. As a result, emergence of resistant organisms is commonplace. Antibiotic prophylaxis in surgery consists of a short antibiotic course given immediately before the procedure in order to prevent development of a surgical site infection. The basic principle of prophylaxis is to maintain effective concentrations of an antibiotic active against the commonest pathogens during the entire surgery.

Materials and methods: We prospectively investigated 427 urologic surgery cases in our department between August 2008 and September 2009 (Group1). As reference cases, we retrospectively reviewed 966 patients who underwent urologic surgery between May 2004 and May 2008 (Group 2) who were administered antibiotics without any restriction. Prophylactic antibiotics such as cefazolin were administered intravenously according to our protocol. Postoperative body temperature, peripheral white blood cell counts, urinalysis, and urine culture were checked.

Results: To judge perioperative infections, wound condition and general condition were evaluated in terms of surgical site infection, as well as remote infection and urinary tract infection, up to postoperative day 30. Surgical site infection was defined as the presence of swelling, tenderness, redness, or drainage of pus from the wound, superficially or deeply. Remote infection was defined as occurrence of pneumonia, sepsis, or urinary tract infection. Perioperative infection rates (for surgical site and remote infection) in Group 1 and Group 2 were nine of 427 (2.6\%) and 24 of 966 (2.5\%), respectively. Surgical site infection rates of categories A and B in Group 1 were 0 and two $(0.86 \%)$, respectively, while those in Group 2 were 0 and five $(0.92 \%)$, respectively. There was no significant difference in infection rates in terms of remote infection and surgical site infection between Group 1 and Group 2 $(P=0.670)$. The amounts, as well as the prices, for intravenously administered antibiotics decreased to approximately one quarter.

Conclusion: Our protocol effectively decreased the amount of antibiotics used without increasing perioperative infection rates. Thus, our protocol of prophylactic antibiotic therapy can be recommended as an appropriate method for preventing perioperative infection in urologic surgery.

Keywords: surgical site infection, antibiotic prophylaxis, single dose, urologic surgery

\section{Introduction}

For more than two decades it has been claimed that prophylactic antibiotics are often inappropriately used in a variety of surgical procedures. ${ }^{1}$ Inappropriate antibiotic use increases environmental selection pressure, favoring the emergence of antimicrobialresistant bacteria that can cause surgical site infections, resulting in administration of more antibiotics, an increase in the cost of care, and a prolonged hospital stay. ${ }^{2}$ Surgical 
site infection is defined by the Centers for Disease Control and Prevention as an infection occurring at or near the surgical incision within 30 days of a procedure. ${ }^{3}$ Rates of surgical site infection are emerging as the leading indicator of quality in surgery. Attention to surgical site infection as a surrogate of quality, combined with the growing problem of antibioticinduced resistance, has brought the issue of prophylaxis to center stage. Antimicrobial prophylaxis is the periprocedural systemic administration of an antimicrobial agent intended to reduce the risk of local and systemic postprocedural infections. The potential benefit of antimicrobial prophylaxis is determined by patient-related factors (ability of the host to respond to bacterial invasion), procedural factors (likelihood of bacterial invasion at the operative site), and the potential morbidity of infection. Antimicrobial prophylaxis is recommended only when the potential benefit outweighs the risks and anticipated costs (including expense of agent and administration, risk of allergic reactions or other adverse effects, and induction of bacterial resistance). The prophylactic agent should be effective against organisms characteristic of the operative site. Cost, safety, and convenience of the agent should also be considered. The duration of antimicrobial prophylaxis should extend throughout the period when bacterial invasion is facilitated and/or likely to establish an infection. ${ }^{4}$ There have been many reports and a comprehensive review on the prevention of surgical site infection and the use of antimicrobial prophylaxis in general surgery. ${ }^{5}$ However, in urologic surgery, only a few papers have been published, except for those on transurethral prostatectomy. ${ }^{6}$ The aim of the present study was to assess whether our antimicrobial prophylaxis protocol, which was designed to decrease the use of antibiotics as well as perioperative infection rates, was appropriate in urologic surgery in Iran.

\section{Materials and methods}

We prospectively investigated 427 patients who underwent urologic procedures at our center from August 2008 to September 2009 (Group 1). A total of 1393 cases were analyzed, consisting of 427 cases in Group 1 and 966 cases in Group 2. The mean age of the two groups were 47 years (range 1-89) for Group 1 and 51.5 years (range 4-111) for Group 2. The number of patients in categories A and B were 196 and 231 in Group 1, and 428 and 538 in Group 2, respectively (Table 3 ). In each category, there were no statistically significant differences between Group A and Group B in terms of clinical background including age, gender, body mass index, hemoglobin, smoking, operation time, and bleeding (Table 1). We classified our surgical operations into two categories according to invasiveness and contamination level, ie, category A (clean and less invasive surgery, eg, endoscopic surgery) and category B (clean invasive surgery or clean contaminated surgery, Table 5). Patients with systemic or local signs and symptoms of infection were excluded from the study. Urinalysis and urine culture were performed for all of our eligible cases. Cases of positive or suspicious urine culture before operation were also excluded. Antibiotics were administered intravenously according to our protocol, ie, cefazolin during the induction of anesthesia for both categories. All of the endourologic cases, except for cystoscopy, had insertion of an indwelling Foley catheter for at least 24 hours postoperatively. In addition, we inserted an indwelling Foley catheter postoperatively for cases of open simple or radical prostatectomy, open pyelolithotomy or nephrolithotomy, radical nephrectomy or partial nephrectomy, and ureterolithotomy. Postoperative oral antibiotics were not initially administered. The occurrence of surgical site infection and remote infection in Group 1 was compared with the retrospectively reviewed reference group of 966 cases who underwent urologic surgery with uncontrolled administration of antibiotics from March 2006 to April 2008 (Group 2). We also analyzed risk factors for surgical site infection or remote infection in Group 1, including preoperative patient factors (age, gender, body mass index, smoking status, diabetes mellitus, hypertension, and hemoglobin concentration), and intraoperative conditions (duration of surgery and amount of bleeding). In Group 2, intravenous cefazolin was given for 24-48 hours after surgery and in Group 1 only a single-dose intravenous cefazolin dose was given at the time of operation. In all cases, including both groups, the preoperative hospital stay was less than 24 hours. The new one-dose protocol required that cefazolin $1 \mathrm{~g}$ would be given at induction of anesthesia. No doses would be given after the end of surgery. All patients were visited on postoperative days 2 and 4. If local or systemic signs or symptoms of infection, including fever, tenderness, and/or swelling at the incision site were detected, an appropriate oral or intravenous antibiotic was commenced. In addition, complete blood count, urinalysis, and urine culture were performed 24 hours after operation and 48 hours after Foley catheter removal. The approximate cost

Table I Number of adult and pediatric cases

\begin{tabular}{llll}
\hline & Group I & Group 2 & Total \\
\hline Adult & 391 & 866 & 1257 \\
Pediatric & 36 & 100 & 136 \\
\hline
\end{tabular}


of a cefazolin $1 \mathrm{~g}$ vial was $1 \mathrm{USD}$. When a patient showed signs of systemic infection, ie, body temperature $\geq 38^{\circ} \mathrm{C}$, a white blood cell count $>12,000 / \mathrm{mm},{ }^{3}$ or localized signs or symptoms including pain, swelling, redness, wound drainage, and tenderness, treatment using another appropriate antibiotic was started, and the case judged as a failure to prevent perioperative infection.

\section{Statistical analysis}

SPSS software (version 16; SPSS Inc., Chicago, IL) was used for the statistical analysis. A $P$ value of less than 0.05 was considered significant.

\section{Results}

Perioperative infections, including surgical site infection and remote infection, were observed up to 30 days postoperation. We primarily judged perioperative infections from the wound condition and general condition at the second or fourth day after operation. Perioperative infection, including both surgical site infection and remote infection, occurred in nine of 427 patients (2.6\%) in Group 1 and in 24 of 966 (2.5\%) in Group 2 (Table 4). There was no statistically significant difference in perioperative infection rates between Group 1 and Group $2(P=0.670)$. Rates of surgical site infection in Group 1 were 0 and $2(0.86 \%)$ in categories A and B, respectively, while those in Group 2 were 0 and $5(0.92 \%)$, respectively. Again, there was no statistically significant difference in the rate of surgical site infection in each category between Group A and Group B ( $P=0.670$ and $P=0.667$, respectively, Table 2). In categories A and B, the amount of intravenously administered antibiotics per patient in Group 1 was significantly smaller than that in Group B. Thus, the average price for intravenously administered antibiotics decreased to approximately one quarter (1 USD for Group 1 versus 4 USD for Group 2) and the average price for oral antibiotics decreased to approximately one-fifth (0.5 USD for Group 1 and 2 USD for Group 2). No significant differences were found between the single-dose group and the two-day group in terms of total surgical site infection, superficial incisional surgical site infection, deep incisional surgical site infection, febrile urinary tract infections, or pneumonia. In both groups, underlying conditions, such as diabetes, did not have an influence on the incidence of postoperative complications.

\section{Discussion}

Surgical site infection and urinary tract infection are a common cause of patient morbidity. Surgical site infections complicate up to $5 \%$ of clean extra-abdominal operations and up to $20 \%$ of intra-abdominal procedures. ${ }^{7}$ There are many potential factors to consider in choosing an appropriate perioperative antibiotic regimen. These considerations include the infection rate at both the surgical site and at remote sites, the potential development of antimicrobial resistance, cost, and the potential for adverse reactions to the antibiotic. Surgical site infections increase morbidity and mortality and can incur considerable costs to an already overwhelmed health care system. Surgical antimicrobial prophylaxis has been shown to reduce the incidence of postoperative wound infections in many randomized clinical trials. The drug chosen should be active against the pathogens most commonly associated with wound infections following the specific procedure and against the pathogens endogenous to the region of the body being operated on, ${ }^{8}$ but need not be active against every potential pathogen. ${ }^{9}$ The prophylactic dose should never be smaller than the standard therapeutic dose of the drug. It is reasonable to use a dose in the upper therapeutic range (eg, 1-2 g of cefazolin or cefotetan for adults and $30-40 \mathrm{mg} / \mathrm{kg}$ for children).

Infection can be prevented when effective concentrations are present in the blood and tissues during and shortly after

Table 2 Clinical background and data for Group I and Group 2

\begin{tabular}{|c|c|c|c|c|}
\hline & \multicolumn{2}{|l|}{ Group I } & \multicolumn{2}{|l|}{ Group 2} \\
\hline & Category A & Category B & Category A & Category B \\
\hline Cases (n) & 196 & 231 & 428 & 538 \\
\hline Median age & 44.2 & 53.5 & 47.5 & 51.7 \\
\hline BMI, median (range) & $22(18-27)$ & $23.5(19-31)$ & $21(18.5-28)$ & $24(18-30)$ \\
\hline Sex, female/male & $17 / 179$ & $19 / 212$ & $41 / 387$ & $80 / 458$ \\
\hline Median $\mathrm{Hb}$ (range, g/dL) & 13 & 13.5 & 14 & 13.8 \\
\hline Smoking, yes/no & $57 / 139$ & $64 / 167$ & $132 / 296$ & $161 / 377$ \\
\hline Hypertension & $28 / 168$ & $42 / 189$ & $87 / 341$ & I | 4/424 \\
\hline Diabetes mellitus & $14 / 182$ & $51 / 180$ & $47 / 381$ & $92 / 446$ \\
\hline
\end{tabular}

Abbreviations: BMI, body mass index; $\mathrm{Hb}$, hemoglobin. 
Table 3 Major operation types of groups and categories

\begin{tabular}{|c|c|c|c|c|c|}
\hline Category A & Group I & Group 2 & Category B & Group I & Group 2 \\
\hline TURP & 63 & 72 & SOP & 67 & 143 \\
\hline TUL & 74 & 75 & Oss & 31 & 58 \\
\hline TURB & 21 & 31 & Nephrectomy & 11 & 29 \\
\hline IU & 14 & 16 & Varicocelectomy & 53 & 131 \\
\hline PB & 4 & 10 & Herniorrhaphy & 34 & 87 \\
\hline BB & 7 & 5 & Hydrocelectomy & 35 & 69 \\
\hline CLL & 13 & 22 & Open cystolithotomy & 8 & 21 \\
\hline Total & 196 & 231 & Total & 239 & 538 \\
\hline
\end{tabular}

Abbreviations: BB, bladder biopsy; CLL, cystolitholapaxy; IU, internal urethrotomy; OSS, open stone surgery; PB, prostate biopsy; SOP, suprapubic open prostatectomy; TUL, transuretheral lithotripsy; TURB, transurethral resection of bladder; TURP, transurethral resection of prostate.

the surgical procedure. Therefore, antimicrobial prophylaxis should begin just before the operation. Starting earlier is unnecessary and potentially dangerous, and starting later is less effective. ${ }^{9}$ Current information indicates that additional intraoperative doses of an antimicrobial agent should be given at intervals of one- or two-fold the half-life of the drug so that adequate levels are maintained throughout the operation. ${ }^{10}$ Because the half-life of almost all antibiotics is $0.7-1.5$ hours, it is necessary to administer antibiotics again when the operation time is more than three hours. ${ }^{11}$ Supplementary doses are indicated in cases where blood loss is greater than $1500 \mathrm{cc}$.

Misuse of antibiotics is not harmless. Increasing adverse effects, bacterial resistance, and costs are commonly associated with antibiotic use. To our knowledge, no one has demonstrated an increase in adverse effects using surgical antibiotic prophylaxis. Many risk factors have been reported, such as age, nutritional status, diabetes mellitus, smoking, and obesity, ${ }^{12}$ as well as coexistent infections at a remote body site, colonization with microorganisms, altered immune response, length of preoperative stay, transfusion, preoperative hair removal, antimicrobial prophylaxis, operating room, surgical attire and drapes, and surgical technique.

Taken together, we classified surgical procedures according to invasiveness, contamination levels (Table 5), and antimicrobial prophylaxis schedule, including timing, period, and the selection of antimicrobial agents designed according to each category. Because the targets are not only Gram-positive but also Gram-negative bacteria in category B, first- or second-generation cephems for skin incisions are recommended. ${ }^{8}$

We believe that our protocol was very simple for medical staff to implement. Most importantly, there were no significant differences in the rates of surgical site infection as well as remote infection in each category between the two groups, in spite of a decrease in the amount of antimicrobial prophylaxis. In a study by Briffaux et al there was no significant difference between two antibiotic prophylaxis regimens (single-dose or three-day) for patients undergoing transrectal ultrasound-guided biopsies. ${ }^{13}$ In a study by Zomorrodi and Buhluli ${ }^{14}$, there was no difference between 1 day and 7 days antibiotic prophylaxis in donor nephrectomy cases. In a study by Trinchieri et al antimicrobial prophylaxis according to European Association of Urology guidelines together with active surveillance seemed to be adequate to prevent symptomatic/febrile genitourinary infections, as well as serious wound infections, in the majority of patients. ${ }^{15}$

An appealing argument for decreasing antibiotic usage may involve cost. Our study showed that adjusting 24-hour prophylaxis to one dose-prophylaxis reduces costs without increasing infection rates, and results in monthly cost savings. Importantly, our savings are not restricted to decreasing two to three doses per surgery, considering that overuse of antibiotics may be much more expensive than the cost of the drug itself. Resistant organisms, potential allergic reactions, and other adverse events related to antibiotic use will certainly cost more than the 3 USD per

Table 4 Infection rates in Group I and Group 2

\begin{tabular}{|c|c|c|c|c|c|c|c|c|}
\hline \multicolumn{5}{|l|}{ Group I } & \multicolumn{4}{|c|}{ Group 2} \\
\hline Category & $\mathbf{n}$ & SSI (\%) & RI (\%) & Total (\%) & $\mathbf{n}$ & SSI (\%) & RI (\%) & Total (\%) \\
\hline A & 196 & 0 & $3(1.5)$ & $3(1.5)$ & 428 & 0 & $9(2)$ & $9(2)$ \\
\hline B & 231 & $2(0.86)$ & $4(1.7)$ & $6(2.5)$ & 538 & $5(0.92)$ & $10(1.8)$ & I5 (2.72) \\
\hline Total & 427 & $2(0.46)$ & $5(1.2)$ & $9(2.6)$ & 966 & $5(0.5)$ & $19(2)$ & $24(2.5)$ \\
\hline
\end{tabular}

Abbreviations: SSI, surgical site infection; RI, remote infection. 
Table 5 Category classification of typical urologic surgery

\begin{tabular}{|c|c|c|}
\hline Type & Definition & Procedures \\
\hline \multirow[t]{2}{*}{ Category A } & Clean, less invasive surgery, & \\
\hline & Endoscopic/laparoscopic surgery & \\
\hline \multirow[t]{2}{*}{ Open surgery } & & Varicocelectomy, orchidopexy, \\
\hline & & Hydrocelectomy, orchiectomy, etc \\
\hline Laparoscopic surgery & & Adrenalectomy, nephrectomy, etc \\
\hline Endoscopic surgery & & TURB, TURP, TUL, etc \\
\hline \multirow[t]{2}{*}{ Category B } & Clean, invasive surgery, clean-contaminated surgery, & \\
\hline & a part of endoscopic and laparoscopic surgery & \\
\hline \multirow[t]{4}{*}{ Open surgery } & & Nephrectomy, nephroureterectomy, \\
\hline & & partial nephrectomy, pyeloplasty, total or \\
\hline & & partial cystectomy, open prostatectomy, \\
\hline & & radical prostatectomy, RPLND, etc. \\
\hline \multirow[t]{2}{*}{ Laparoscopic surgery } & & Nephroureterectomy, partial \\
\hline & & nephrectomy, radical prostatectomy \\
\hline Endoscopic surgery & & PNL, endopyelotomy, etc \\
\hline Category C & Urinary tract diversion using the intestine & $\begin{array}{l}\text { Total cystectomy (ileal conduit, } \\
\text { neobladder), bladder augmentation, etc }\end{array}$ \\
\hline Category D & Dirty or infected surgery & Categories A, B and C with infection \\
\hline
\end{tabular}

Abbreviations: PNL, percutaneous nephrolithotomy; TURP, transurethral resection of prostate; RPLND, retroperitoneal lymph node dissection; TUL, transurethral ureterolithotripsy.

vial of cefazolin. In countries with limited resources, such as Iran, even modest savings can have an impact. Evans et $\mathrm{al}^{16}$ reported recently that the median cost of treating a resistant Gram-negative infection in a surgical intensive care unit was 80,500 USD compared with the cost of treating a patient with a sensitive Gram-negative infection, which was 29,604 USD $(P<0.001)$. These authors have concluded that "... efforts to control overuse of antibiotics should be pursued". ${ }^{17}$

\section{Conclusion}

A single-dose antimicrobial prophylaxis regimen was effective for prevention of perioperative infections, including surgical site infection, urinary tract infection, and remote infection in endoscopic-instrumental, clean, and cleancontaminated surgical procedures in urologic patients. We have demonstrated that single-dose prophylaxis is feasible. To the best of our knowledge, this is the first reported study from Iran to evaluate the role of antimicrobial prophylaxis in urologic surgery. In the current era of restricted hospital budgets, one-dose prophylaxis may provide a way to improve performance by lowering costs.

\section{Disclosure}

The authors report no conflicts of interest in this work. We acknowledge general practitioners from Islamic Azad and Qom University of Medical Sciences, Ali Safari, Amir Hossein Shokravi, and Seiied Ali Zaargar, for their data collection and other logistic support.

\section{References}

1. Shapiro M, Townsend TR, Rosner B, Kass EH. Use of antimicrobial drugs in general hospitals: Patterns of prophylaxis. N Engl J Med. 1979; 301(7):351-355.

2. McGowan JE Jr. Cost and benefit of perioperative antimicrobial prophylaxis: Methods for economic analysis. Rev Infect Dis. 1991;13 Suppl 10:S879-S889.

3. Mangram AJ, Horan TC, Pearson ML, et al. Guidelines for prevention of surgical site infection. Hospital Infection Control Practices Advisory Committee. Infect Control Hosp Epidemiol. 1999;(20):250-278.

4. Schmidt AH. Are single-dose preoperative antibiotic regimens really appropriate? Arch Surg. 2007;142(6):576-577.

5. Auerbach AD. Prevention of surgical site infections. In: Shonjania KG, Duncan BW, McDonald KM, Wachter RM, editors. Making Health Care Safer: A Critical Analysis of Patient Safety Practices. Rockville, MD: AHRQ Publication; 2001.

6. Qvist N, Christiansen HM, Ehlers D. Prophylactic antibiotics in transurethral prostatectomy. Urol Res. 1984;12(6):275-277.

7. Bratzler DW, Houck PM. Surgical Infection Prevention Guideline Writers Workgroup. Antimicrobial prophylaxis for surgery, an advisory statement from the National Surgical Infection Prevention Project. Am J Surg. 2005;189(4):395-404.

8. Sojun K, Akito T, Satoshi I, et al. Assessment of a protocol for prophylactic antibiotics to prevent perioperative infection in urological surgery: A preliminary study. Int $J$ Urol. 2004;11(6):355-363.

9. Geroulanos S, Marathias K, Krians J, Kadas B. Cephalosporins in surgical prophylaxis. J Chemother. 2001;13 Spec No 1(1):23-26.

10. Dellinger EP, Gross PA, Barrett TL, et al. Quality standard for antimicrobial prophylaxis in surgical procedures. Clin Infect Dis. 1994; 18(3):422-427.

11. Galandiuk S, Polk HC Jr, Jagelman DG, Fazio VW. Re-emphasis of priorities in surgical antibiotic prophylaxis. Surg Gynecol Obstet. 1989; 169(3):219-222.

12. Waisbren E, Rosen H, Bader AM, Lipsitz SR, Rogers SO Jr, Eriksson E. Percent body fat and prediction of surgical site infection. J Am Coll Surg. 2010;210(4):381-389.

13. Briffaux R, Merlet B, Normand G, et al. Short or long schemes of antibiotic prophylaxis for prostate biopsy. A multicentre prospective randomised study. Prog Urol. 2009; 19(1):39-46. 
14. Zomorrodi A, Buhluli A. Is antibiotic usage necessary after donor nephrectomy? A single center experience. Saudi J Kidney Dis Transpl. 2008;19(2):200-205.

15. Trinchieri A, Paparella S, Cappoli S, et al. Prospective assessment of the efficacy of the EAU guidelines for the prevention of nosocomial acquired infections after genitourinary surgery in a district hospital. Arch Ital Urol Androl. 2009;81(1):46-50.
16. Evans HL, Lefrak SN, Lyman J, et al. Cost of Gram-negative resistance. Crit Care Med. 2007;35(1):89-95.

17. Wolf JS Jr, Bennett CJ, Dmochowski RR, Hollenbeck BK, Pearle MS, Schaeffer AJ. Best practice policy statement on urologic surgery antimicrobial prophylaxis. J Urol. 2008;179(4):1379-1390.

\section{Publish your work in this journal}

Therapeutics and Clinical Risk Management is an international, peerreviewed journal of clinical therapeutics and risk management, focusing on concise rapid reporting of clinical studies in all therapeutic areas, outcomes, safety, and programs for the effective, safe, and sustained use of medicines. This journal is indexed on PubMed Central, CAS,
EMBase, Scopus and the Elsevier Bibliographic databases. The manuscript management system is completely online and includes a very quick and fair peer-review system, which is all easy to use. Visit http://www.dovepress.com/testimonials.php to read real quotes from published authors.

Submit your manuscript here: http://www.dovepress.com/therapeutics-and-clinical-risk-management-journal 